\title{
Soliton compression in short lengths of microstructured fibres
}

\author{
P. Horak, M. L. V. Tse, F. Poletti, and D. J. Richardson \\ Optoelectronics Research Centre, University of Southampton, Southampton SO17 1BJ, United Kingdom
}

Compression of soliton pulses propagating in optical fibers with decreasing dispersion is a well-established technique. Very recently, we have demonstrated the same effect with femtosecond pulses in dispersion-decreasing microstructured holey fibres. ${ }^{1}$ With such short pulses and because of the small core size and thus high nonlinearity possible within holey fibres, compression already occurs at very low soliton energies and over short lengths of fibre. Short fibre lengths are also required because of higher propagation losses in holey fibres which counteract compression. Here we investigate theoretically the minimum fibre length necessary to achieve high compression factors.

Figure 1(a) shows the dispersion and effective area of standard hexagonal holey fibres at $1.55 \mu \mathrm{m}$ wavelength versus geometrical parameters. Specifically, we investigate fibres with dispersion $\mathrm{D}=25 \mathrm{ps} / \mathrm{nm} / \mathrm{km}$ and effective area $A_{\text {eff }}=70 \mu \mathrm{m}^{2}$ at the START point and $D=5 \mathrm{ps} / \mathrm{nm} / \mathrm{km}, A_{\text {eff }}=30 \mu \mathrm{m}^{2}$ at the END point. Such fibres can be fabricated with different variation of parameters along the fibre length, as e.g. indicated by Paths 1, 2, 3 in Fig. 1(a), and with different lengths.

(a)

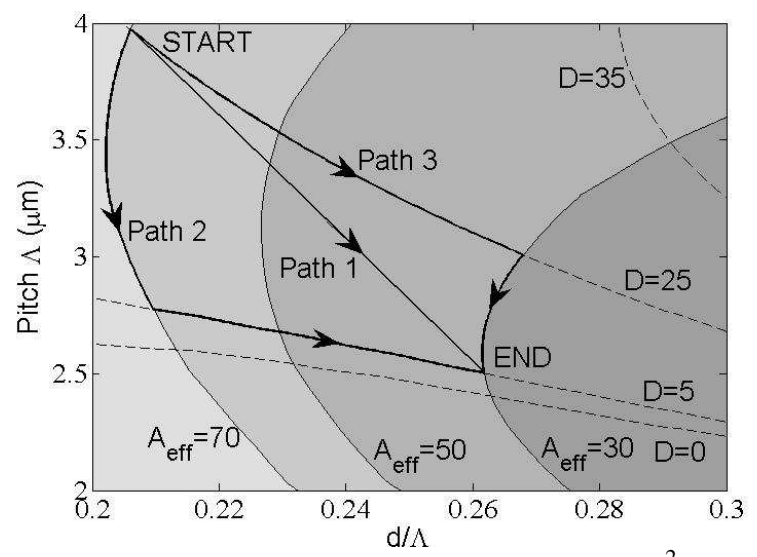

(b)

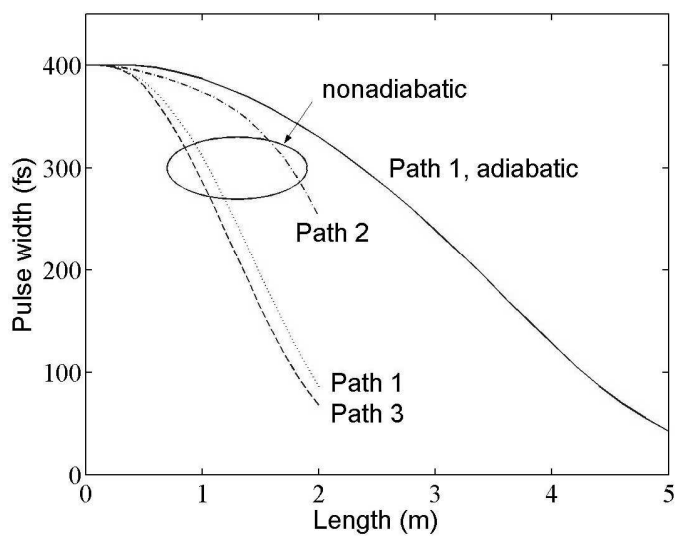

Fig. 1 (a) Contour map for effective area $\left(\mu^{2}\right)$ and dispersion $(\mathrm{ps} / \mathrm{nm} / \mathrm{km})$ versus $\Lambda$ and $\mathrm{d} / \Lambda$ for holey fibers of hexagonal geometry at $1.55 \mu \mathrm{m}$ wavelength. (b) Pulse compression along Paths 1, 2, 3 under optimised adiabatic and nonadiabatic conditions.

1. Adiabatic compression. For slowly varying parameters, soliton compression occurs adiabatically and the soliton width is proportional to $\mathrm{D}$ and $\mathrm{A}_{\text {eff }}$, thus predicting compression by a factor of $\sim 12$ along any of the Paths 1-3, in agreement with numerical simulations of fibres tens of metres long. The shortest length over which adiabaticity can be achieved requires constant effective gain along the fibre. ${ }^{2}$ Simulations show that fibres as short as $5 \mathrm{~m}$ allow for pulse compression by a factor of 10, see Fig. 1(b), thus indicating near-adiabatic conditions.

2. Nonadiabatic compression. For even shorter fibre lengths, pulse propagation is no longer adiabatic. However, significant compression can still be observed. As an example, Figure 1(b) shows compression over only $2 \mathrm{~m}$ of fibre following Paths 1-3. Here, achievable compression varies significantly among different paths. The shortest pulses are found for Path 3 where for the first part of the fibre only $A_{\text {eff }}$ is decreased at constant $D$ and for the second part of the fibre only $\mathrm{D}$ is decreased at constant $\mathrm{A}_{\text {eff. }}$. In this latter case, compression of $400 \mathrm{fs}$ pulses down to $65 \mathrm{fs}$ is observed.

In conclusion, we have investigated compression of femtosecond solitons in holey fibres of decreasing dispersion and effective mode area. Best compression is obtained in the adiabatic propagation regime where the effective gain is small and constant along the fibre. For shorter fibre lengths, compression is nonadiabatic, but high compression factors can still be achieved by careful optimisation of $\mathrm{D}$ and $\mathrm{A}_{\text {eff }}$ along the fibre. For the chosen parameters, we find compression factors of $\sim 10$ in the near-adiabatic regime over $5 \mathrm{~m}$ of fibre, and $\sim 6$ in the nonadiabatic regime over $2 \mathrm{~m}$.

\section{References}

1. M. L. V. Tse et al., "Pulse compression at $1.06 \mu \mathrm{m}$ in dispersion-decreasing holey fibers," Opt. Lett. 31, 3504 (2006). 2. V. A. Bogatyrev et al., "A single-mode fibre with chromatic dispersion varying along the length," J. Lightwave Technol. 9, 561 (1991). 